\title{
Ventilation and Ventilatory Pattern During Sleep in Aborted Sudden Infant Death Syndrome
}

\author{
GABRIEL G. HADDAD, ${ }^{(32)}$ HEDI L. LEISTNER, TZE L. LAI, AND ROBERT B. MELLINS \\ Departments of Pediatrics (Pulmonary Division) and Mathematical Statistics, Columbia University and Babies \\ Hospital, New York, New York, USA
}

\section{Summary}

To assess ventilatory control during sleep in infants at risk for the sudden infant death syndrome (SIDS), we made serial measurements of resting tidal volume $(\mathrm{Vt})$, respiratory cycle time (Ttot), and the ventilatory changes resulting from inhalation of $2 \%$ $\mathrm{CO}_{2}$ in aborted SIDS infants in rapid eye movement and quiet sleep and compared them to a group of normal infants during the first 4 months of life. Ventilation was measured by the barometric method, and sleep was staged using electroencephalogram, electrooculogram, and electromyogram and behavioral criteria.

Although resting instantaneous minute ventilation (Vt/Ttot) was virtually the same in both groups of infants, $\mathrm{Vt}$ tended to be smaller (by up to $50 \%$ in the first $\mathbf{2}$ months) and Ttot tended to be shorter in aborted SIDS than in normal infants in both rapid eye movement and quiet sleep. The increase in the mean $\mathrm{Vt} / \mathrm{T}$ tot with $2 \% \mathrm{CO}_{2}$ is greater by about 5 to $20 \%$ in aborted SIDS than in normal infants at 3 and 4 months of age in both sleep states. These findings, together with our previous findings that aborted SIDS infants have an increase in heart rate and a shortening of the QT interval, provide indirect evidence that infants at high risk for SIDS may have increased sympathoadrenal activity.

\section{Speculation}

We speculate that cardiorespiratory collapse in SIDS is the result of an imposition of a stress, such as transient hypoxia, on an infant with a developmental abnormality in the sympathetic nervous system in whom the maturation of protective mechanisms against such an abnormality is not yet fully completed.

Although it has generally been assumed that there is a maturational defect in the cardiorespiratory control system of infants with the sudden infant death syndrome (SIDS) $(27,28)$, to our knowledge, no ventilatory studies have been performed on aborted SIDS infants to see whether these infants have abnormal maturational changes in minute ventilation or ventilatory pattern. Therefore, we studied resting minute ventilation and ventilatory pattern serially in the first 4 months of life in aborted SIDS infants and compared them to those in age-matched normal infants. To determine whether $\mathrm{CO}_{2}$ inhalation induces different ventilatory changes in aborted SIDS than in normal infants, we also compared ventilation and ventilatory pattern obtained during $\mathrm{CO}_{2}$ inhalation to those obtained during ambient air breathing in both populations in the same age period. Because facial contact may disturb the level as well as the pattern of ventilation $(3,12)$, we utilized the noninvasive barometric method (10) as modified in our laboratory $(11,14)$ to measure ventilation. Because death in SIDS infants is presumed to occur during sleep $(27,28)$ we studied all ventilatory parameters in both groups of infants during REM and quiet sleep.
SUBJECTS AND METHODS

PATIENT POPULATION

Nineteen normal infants and 12 infants with one or more episodes of aborted SIDS were studied. There were 12 male and seven female infants in the normal group and five male and seven female infants in the aborted SIDS group. Four aborted SIDS infants had siblings, and two had cousins who died of SIDS. Except for 1 aborted SIDS infant who was born at $35 \mathrm{wk}$ of gestation and two normal infants born at $37 \mathrm{wk}$ of gestation, all infants in both groups were full-term. All pregnancies and deliveries were uncomplicated. Their birth weights ranged between 2700 to $4040 \mathrm{~g}$. There were no significant differences in the means of the two groups in birth weight or subsequent growth.

We defined an aborted SIDS infant as an apparently healthy infant who has experienced an episode of unexplained apnea with cyanosis or pallor requiring mouth to mouth resuscitation or vigorous physical stimulation for revival. The mean age of the infants at the time of the first aborted SIDS episode was 2 months, with a range of 1 to 4 months. Eight aborted SIDS infants were observed to be asleep shortly before their apneic episode whereas the state of consciousness was unknown in the remaining four. Four of the infants were found limp, four were stiff, and no abnormal muscle tone was noted in the other four infants. Cyanosis was observed in seven of the aborted SIDS infants and pallor in five. Three of the 12 infants had more than one episode of aborted SIDS.

We included in the group of the aborted SIDS infants only infants that had normal physical and detailed neurologic examination and negative laboratory assessment. This assessment included blood counts, multiple blood chemistries (serum glucose, sodium, potassium, chloride, calcium, magnesium, and blood urea nitrogen), acid-base and blood gas analysis, chest x-ray, upper gastrointestinal study with barium swallow to exclude marked gastroesophageal reflux, 12-lead electrocardiogram (ECG), 12 to 24-hr continuous ECG monitoring on tape, and electroencephalogram. Conventional 12-lead ECGs performed at monthly intervals in the first 4 months of life were normal in all infants. These infants are now 6 to 48 months old and have had normal growth and development.

\section{PHYSIOLOGIC STUDIES}

Our methods have been described in detail elsewhere $(14,16$, 21). In brief, the aborted SIDS infants were first studied within 1 to 2 wk after the aborted SIDS episode. Subsequently, ventilatory measurements were serially performed on their monthly birthdays through 4 months of age. The normal infants were studied at 1, 2, 3 , and 4 months of age. Informed written consent was obtained from the parents of all infants. Because of imperfect parental compliance and the different ages of presentation in the aborted SIDS infants, the number of normal infants studied at each age varied from 14 to 18 and that of aborted SIDS infants from 3 to 
11 with an average of 17 and 7, respectively. In addition, because several of these infants woke up before $\mathrm{CO}_{2}$ was administered, it was not possible to study the ventilatory changes induced by $\mathrm{CO}_{2}$ in all infants. The average total sleep time in a study was $135 \mathrm{~min}$ with a range of 80 to $210 \mathrm{~min}$. The pre- $\mathrm{CO}_{2}$ period averaged about $70 \mathrm{~min}$, and about an equal time was spent in the $\mathrm{CO}_{2}$ sleep period.

Polygraphic recordings were coded into rapid eye movement (REM) and quiet sleep using a bipolar C4-Al electroencephalogram, a bipolar electrooculogram, and a bipolar submental electromyogram (1). A behavioral code that described the infant's somatic activities during sleep (9) was used throughout each study.

Respiration was recorded wholly noninvasively using the barometric method $(11,14,16)$. The techniques utilized for data acquisition have been detailed elsewhere (14). In brief, the respiratory waveform was digitized on-line at $25 \mathrm{~Hz}$ and stored on disk for analysis with the aid of a minicomputer. Tidal volume $(\mathrm{Vt})$, inspiratory time (Ti), expiratory time (Te), and total respiratory cycle time (Ttot) were measured. In addition, we calculated the instantaneous minute ventilation (Vt/Ttot). In each study, after baseline ventilatory measurements, $2 \% \mathrm{CO}_{2}$ was continuously inspired for about $1 \mathrm{hr}$. Two percent $\mathrm{CO}_{2}$ was chosen because in preliminary studies $4 \%$ frequently aroused infants from sleep. To increase the $\mathrm{CO}_{2}$ concentration to $2 \%$ in the chamber, a calculated volume of $80 \% \mathrm{CO}_{2}$ in $\mathrm{O}_{2}$ was introduced in the chamber. The $\mathrm{CO}_{2}$ concentration in the chamber was kept between 1.90 and $2.10 \%$ during the $\mathrm{CO}_{2}$ challenge period. Because $\mathrm{CO}_{2}$ concentration in the chamber did not reach equilibrium for about 2 to 3 min and because we wished to study the ventilatory change after it reached its maximum (16), we did not include the first $5 \mathrm{~min}$ after adding $\mathrm{CO}_{2}$ to the chamber in this analysis.

\section{STATISTICAL ANALYSIS}

The results of the present investigation were based on the analysis of all the artifact-free breaths which amounted to 5,000 to 10,000 breaths per study. In every study, each ventilatory parameter for each sleep state was calculated as the median of all the observed values in that sleep state before the administration of $\mathrm{CO}_{2}$ and during $\mathrm{CO}_{2}$ breathing. Because the variances of the two populations (normal and aborted SIDS) were quite different in many cases, we performed the Welch test $(6,21)$ instead of the unpaired $t$ test to evaluate statistical significance in the differences between the means of the two groups of infants at each age and sleep state. Differences in the means were considered significant if $P<0.05$.

The above conventional approach compares the means of the aborted SIDS population and the population of normal infants at each age. In general, when the variances of two populations are not equal or when the distributions of the two populations are skewed, the population with a larger mean may have a relatively low probability of giving a larger sample value than the other population with the smaller mean. Therefore, in addition to the above comparison of means, it is also important to estimate the actual probability $(P[\mathrm{X}>\mathrm{Y} \mid \mathrm{i}])$ that a sample value $\mathrm{X}$ selected at random from one population at the age of $\mathrm{i}$ months exceeds a sample value $\mathrm{Y}$ selected at random from the other population at the same age $(5,30)$. We estimate this probability by using a normalized form of the Mann-Whitney statistic $(5,17,20)$. We then apply the classical Mann-Whitney test (17) to determine whether the estimated probability is significantly above (or below) $1 / 2$ at the 0.05 significance level.

We make use, therefore, of two different statistical techniques for the comparison of each ventilatory variable: (1) the Welch test to see whether there is a significant difference between the means of the two populations; (2) an estimate of the probability that an observation $\mathrm{X}$ selected at random from one population at a given age $\mathrm{i}$ exceeds an observation $\mathrm{Y}$ also selected at random from the other population of infants at the same age; the Mann-Whitney test is applied to see whether this estimated probability is significantly above or below $1 / 2$.

To study the $\mathrm{CO}_{2}$-induced changes in ventilation and ventilatory pattern, we first calculated the difference between the median of all the observed values before the administration of $\mathrm{CO}_{2}$ (baseline median) and that during $\mathrm{CO}_{2}$ breathing for each respiratory parameter in each infant. This difference was then normalized by the baseline median, and this was expressed as median change from baseline in percentage. We then examined the distribution of the values of the median percentage change for each of the two groups of infants at each age and sleep state. These two distributions were compared to each other, and both the Welch test and the Mann-Whitney test were applied to determine whether the two distributions are significantly different from each other.

\section{RESULTS}

\section{RESTING VENTILATION AND VENTILATORY PATTERN}

$V t$. The mean $\mathrm{Vt}$ is smaller in aborted SIDS than in normal infants at all ages; however, the major difference occurs in the first 2 months of life when the percentage difference in the means between normal and aborted SIDS infants reaches almost $50 \%$ (Fig. 1). The differences in means are significant at 1 and 2 months of age in both REM and quiet sleep ( $P<0.05$; Welch test). Because there are no significant differences in weight between aborted SIDS and normal infants at any age, the weight-normalized Vt shows the same differences between normal and aborted SIDS infants.

The actual probability $P[\mathrm{X}>\mathrm{Y} \mid \mathrm{i}]$ that the $\mathrm{Vt}$ of a normal infant (denoted generically by $\mathrm{X}$ ) exceeds the $\mathrm{Vt}$ of an aborted SIDS infant (denoted generically by $\mathrm{Y}$ ) at the age of $\mathrm{i}$ months is also estimated. This probability is significantly above $1 / 2$ at 1 and 2 months of age in both REM and quiet sleep $(P<0.05$; MannWhitney test) and does not fall below $1 / 2$ at the other ages (Table 1). This suggests that aborted SIDS infants tend to breathe with a smaller Vt than do normal infants.

Respiratory Timing (Ttot, Ti, and Te). Except at 3 months of age when the mean Ttot is essentially the same in both groups of infants, the mean Ttot is shorter in aborted SIDS infants at all ages and in both REM and quiet sleep (Fig. 2, top panel); the differences in means are significant at 2 and 4 months of age in both sleep states $(P<0.05$, Welch test $)$. The estimated probability that the Ttot of a normal infant is longer than that of an aborted SIDS infant selected at random is significantly above $1 / 2$ at 2 months in both sleep states and at 4 months in quiet sleep $(P<$ 0.05; Mann Whitney test) and does not fall below $1 / 2$ at the other ages and sleep states (Table 1). This suggests that aborted SIDS infants tend to have a shorter Ttot or tend to breathe at a faster rate than do normal infants. This shortening of Ttot in aborted SIDS infants is in fact due to shortening of both components of Ttot, namely, Ti and Te. Figure 2, middle panel, shows that the

$$
\begin{aligned}
& \text { TIDAL VOLUME } \\
& \text { REM SLEEP }
\end{aligned}
$$

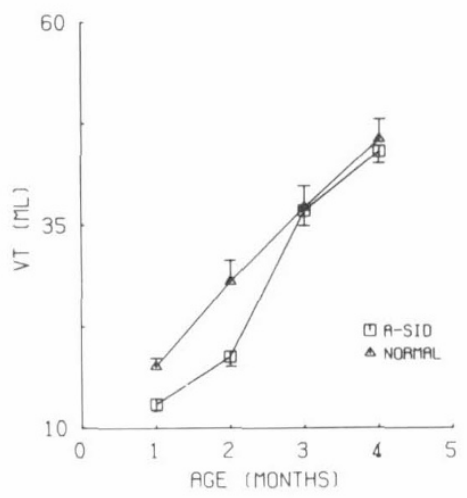

Fig. 1. The mean $\mathrm{V}_{\mathrm{t}}$ is smaller in A-SID infants at each age and is significantly smaller at 1 and 2 months of age in both REM (left) and quiet (right) sleep. Mean \pm I S.E. 
Table 1. Estimated probability that resting Vt, Ttot, or Vt/Ttot, or percentage increase in $\mathrm{Vt} / \mathrm{T}$ tot in response to $\mathrm{CO}_{2}$ of a normal infant chosen at random at a certain age exceeds that of an aborted SIDS infant at the same age in both REM and quiet sleep

\begin{tabular}{cccccc}
\hline $\begin{array}{c}\text { Age } \\
(\mathrm{mos})\end{array}$ & $\begin{array}{c}\text { Sleep } \\
\text { state }\end{array}$ & $\mathrm{Vt}$ & Ttot & $\mathrm{Vt} /$ Ttot & $\begin{array}{c}\text { \% increase } \\
\text { in Vt/Ttot }\end{array}$ \\
\hline $\mathrm{I}$ & $\mathrm{R}^{1}$ & $0.91^{2}$ & 0.64 & 0.48 & $-{ }^{3}$ \\
& $\mathrm{Q}$ & $0.69^{2}$ & 0.59 & 0.47 & - \\
2 & $\mathrm{R}$ & $0.69^{2}$ & $0.76^{2}$ & 0.53 & - \\
& $\mathrm{Q}$ & $0.79^{2}$ & $0.82^{2}$ & 0.52 & - \\
3 & $\mathrm{R}$ & 0.55 & 0.50 & 0.49 & $0.26^{4}$ \\
& $\mathrm{Q}$ & 0.50 & 0.52 & 0.65 & 0.39 \\
4 & $\mathrm{R}$ & 0.58 & $0.67^{2}$ & 0.45 & 0.48 \\
& $\mathrm{Q}$ & 0.56 & 0.63 & 0.44 & $0.19^{4}$
\end{tabular}

\footnotetext{
${ }^{1} \mathrm{R}, \mathrm{REM}$; Q, quiet.

${ }^{2}$ Probability significantly above $1 / 2$ at the 0.05 level (Mann-Whitney test).

${ }^{3}$ Insufficient data for aborted SIDS infants (see text).

${ }^{4}$ Probability significantly below $1 / 2$ at the 0.05 level (Mann-Whitney test).
}

mean $\mathrm{Ti}$ is smaller in the aborted SIDS infants than in normal infants at all ages studied in both REM and quiet sleep; these differences in means are significant at 1,2 , and 4 months in REM sleep and at 2 and 4 months in quiet sleep. Figure 2, bottom panel, shows that, except at 3 months of age, the mean Te is shorter in aborted SIDS than in normal infants at all ages and both sleep states; these differences in means are significant at 1 and 2 months of age in both sleep states.

Instantaneous Vt/Ttot. At each age and sleep state, the mean $\mathrm{Vt} / \mathrm{T}$ tot in aborted SIDS infants is not significantly different from that in normal infants by either the Welch test or the MannWhitney test. Moreover, neither the differences in the mean Vt/ Ttot (Fig. 3) nor the estimated probabilities that an observed Vt/ Ttot from one population is greater than that of another population (Table 1) is consistently in one direction. The weight-normalized $\mathrm{Vt} / \mathrm{T}$ tot in aborted SIDS is not significantly different from that in normal infants.

\section{$\mathrm{CO}_{2}$-INDUCED VENTILATORY CHANGES}

Because we could administer $\mathrm{CO}_{2}$ to only a very small number of aborted SIDS infants at 1 and 2 months of age $(n<3)$, no conclusive inferences could be made from our data as to the sign or magnitude of the $\mathrm{CO}_{2}$-induced changes in the ventilatory variables for aborted SIDS infants at these two ages. We could, however, compare the $\mathrm{CO}_{2}$-induced ventilatory changes between normal and aborted SIDS infants at 3 and 4 months of age more thoroughly.

Instantaneous Vt/Ttot. The aborted SIDS infants showed an increase in $\mathrm{Vt} /$ T tot with $\mathrm{CO}_{2}$ in all cases studied at 3 and 4 months of age. The same was true for normal infants except for few studies; 2 of 16 in REM and 1 of 17 studies in quiet sleep showed actually a decrease in $\mathrm{Vt} / \mathrm{T}$ tot with $\mathrm{CO}_{2}$. The scatter plots in Figure 4 show that the $\mathrm{CO}_{2}$-induced mean percentage increase in $\mathrm{Vt} / \mathrm{Ttot}$ at 3 and 4 months of age is greater in aborted SIDS infants than in normal infants in both REM and quiet sleep. In spite of the relatively small number of infants to whom we could administer $\mathrm{CO}_{2}$ at these two ages, the means are significantly greater in aborted SIDS infants at 3 months of age in REM sleep and 4 months of age in quiet sleep ( $P<0.05$; Welch test) (Fig. 4). As shown in Table 1, the estimated probability that the $\mathrm{CO}_{2}$ induced percentage increase in $\mathrm{Vt} / \mathrm{Ttot}$ of a normal infant is greater than that of an aborted SIDS infant is significantly below $1 / 2$ at these ages and sleep states $(P<0.05$, Mann-Whitney test $)$.

$V t$ and Ttot. At each age and in both REM and quiet sleep,

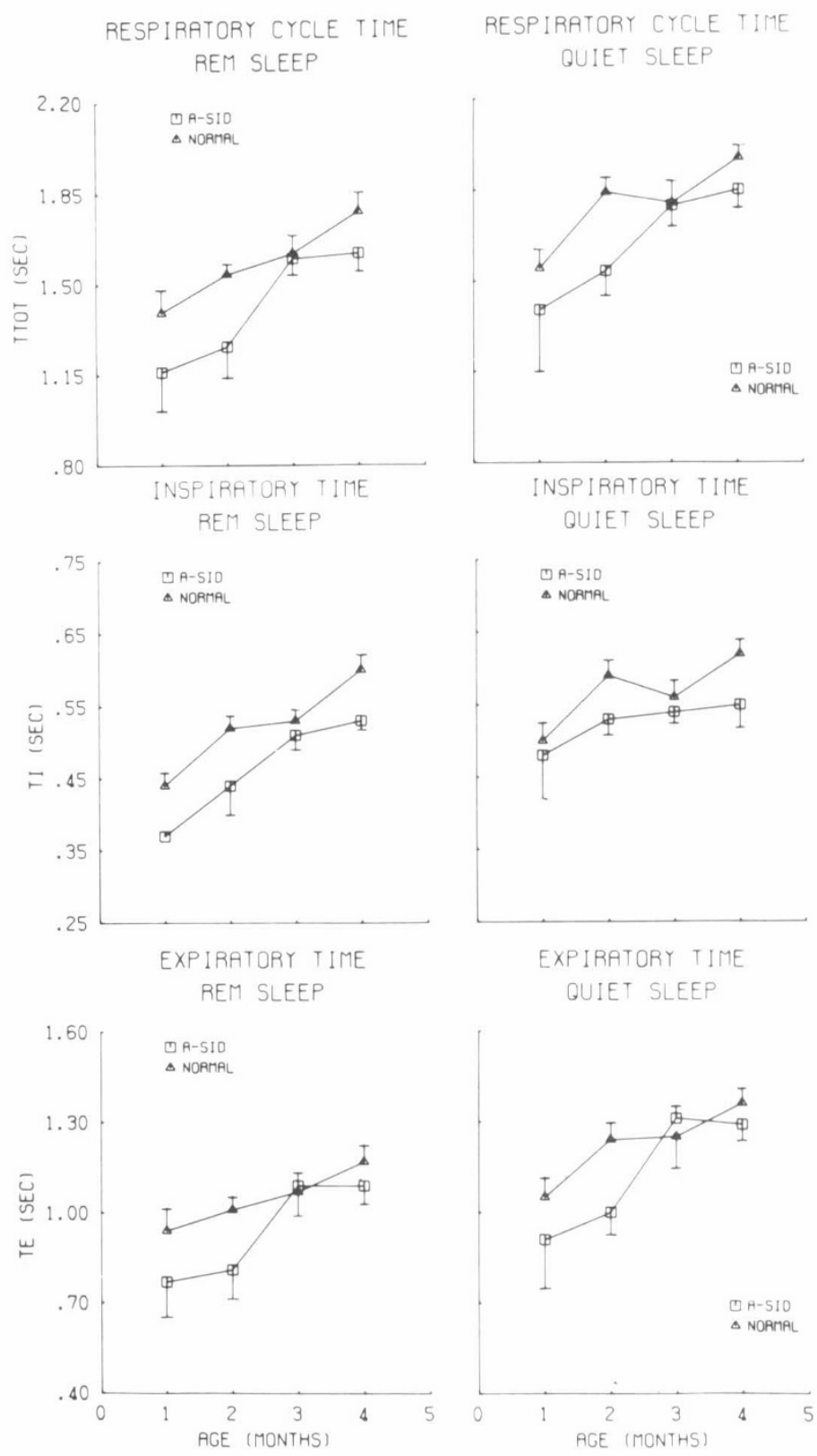

Fig. 2. Top, except at 3 months of age, the mean Ttot is shorter in ASID infants at all ages and is significantly shorter at 2 and 4 months of life in both REM (left) and quiet (right) sleep. Mean \pm 1 S.E. Middle, the mean $\mathrm{Ti}$ is shorter in A-SID infants at all ages and is significantly shorter at 1, 2, and 4 months in REM sleep (left) and at 2 and 4 months in quiet sleep (right). Mean \pm 1 S.E. Bottom, except at 3 months of age, the mean Te is shorter in A-SID infants at all ages and is significantly shorter at 1 and 2 months of age in both REM (left) and quiet (right) sleep. Mean \pm 1 S.E.

there were no clear and consistent differences in the $\mathrm{CO}_{2}$-induced percentages of change in $\mathrm{Vt}$ and $\mathrm{T}$ tot between normal and aborted SIDS infants.

\section{DISCUSSION}

Our results show considerable overlap at each age and sleep state between the population of aborted SIDS infants and the population of normal infants in the ventilatory variables studied. A typical example is the scatter plot of Figure 4. However, in spite of this overlap and the fact that it was only possible to study a relatively small number of aborted SIDS infants, our results show certain clear and consistent tendencies. For example, the mean $\mathrm{Vt}$ in Figure 1 is consistently smaller (although to various degrees) in 
aborted SIDS than in normal infants at all ages and in both REM and quiet sleep, and the differences between the two groups are significant in the first 2 months of age and both sleep states.

In view of the overlap that exists between the two populations, comparisons of the populations in terms of their means is insufficient. Therefore, we also estimate the probability that a sample value $\mathrm{X}$ from one population is greater than a sample value $\mathrm{Y}$ from the other population. This probability can be interpreted as the probability that one population "tends" to exceed the other population for a certain variable, taking into consideration the overlap between them $(17,30)$. It should not, however, be confused with the significance level for determining whether the two populations are statistically different.

\section{$\mathrm{CO}_{2}$-INDUCED VENTILATORY CHANGES IN ABORTED SIDS INFANTS}

This study does not confirm previous results (26) showing that aborted SIDS infants have virtually no increase in minute ventilation in response to $\mathrm{CO}_{2}$. On the contrary, we show that aborted SIDS infants tend to have a larger percentage increase in $\mathrm{Vt} / \mathrm{T}$ tot in response to $\mathrm{CO}_{2}$ when compared to normal infants (Fig. 4; Table 1). There are several possible explanations for the difference between previous results (26) and ours. First, although the infants studied in both investigations are aborted SIDS or near-miss SIDS infants, the study populations may have been different. Second, we studied the ventilatory response to $\mathrm{CO}_{2}$ over a much longer period of time. Third, we studied infants nonin-
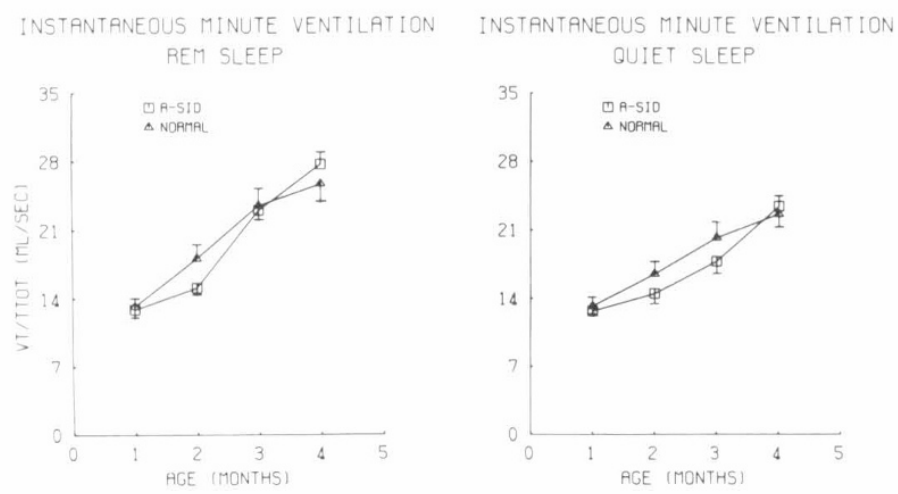

Fig. 3. There is no consistent difference in instantaneous minute ventilation between A-SID and normal infants in both REM (left) and quiet $(r i g h t)$ sleep. Mean \pm I S.E.

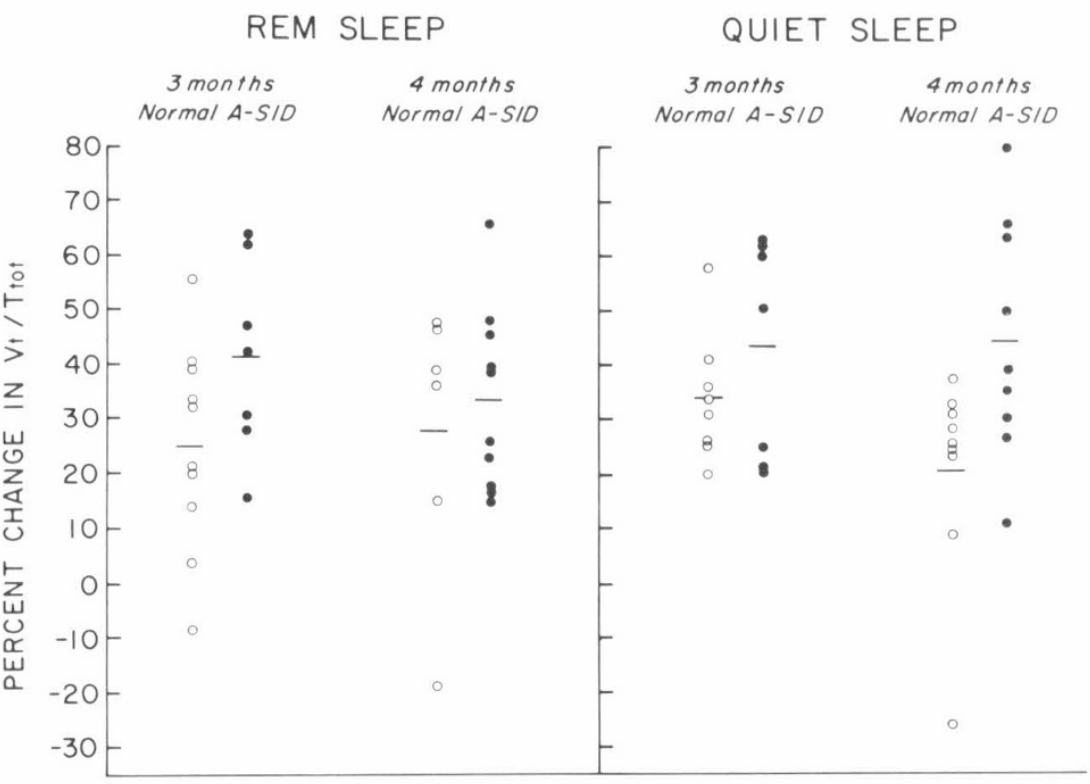

Fig. 4. Scatter diagram showing the percentage change in $\mathrm{Vt} / \mathrm{T}$ tot with $2 \% \mathrm{CO}_{2}$ in both REM and quiet sleep for normal and aborted SIDS infants at 3 and 4 months of age. Greater increase in Vt/Ttot in aborted SIDS infants. Significantly different means are found at 3 months in REM and 4 months in quiet sleep.

vasively by the barometric method. This avoids facial contact, especially with the trigeminal area which, if stimulated, alters breathing and breathing pattern $(3,12)$. Finally, because inhalation of $2 \% \mathrm{CO}_{2}$ may not increase $\mathrm{PaCO}_{2}(2,8)$, whereas $5 \% \mathrm{CO}_{2}$ does (8), we raise the possibility that different receptor systems were stimulated because different concentrations of inhaled $\mathrm{CO}_{2}$ were used in the two studies. Data on animals (22) and man (13) have shown that, in addition to the traditional carotid or medullary chemoreceptors, vagally mediated airway receptors may play an important role in the $\mathrm{CO}_{2}$-induced ventilatory changes. Whether these vagally mediated airway receptors have an increased sensitivity to $\mathrm{CO}_{2}$ in aborted SIDS infants is, at present, speculative.

\section{RESTING VENTILATION IN ABORTED SIDS INFANTS}

Although resting instantaneous minute ventilation in aborted SIDS infants is similar to that in normal infants at each age, the pattern of breathing in aborted SIDS infants differs from that in the normal infants. Aborted SIDS infants tend to have a shorter respiratory cycle time and, therefore, a higher respiratory frequency and a smaller Vt than do normal infants. The causes for this difference in the pattern of breathing in the aborted SIDS infants are not apparent to us at present.

The major difference in the magnitude of $\mathrm{Vt}$ between normal and aborted SIDS infants is in the first 2 months of life. These findings raise two questions: (1) do aborted SIDS infants hypoventilate in the first 2 months of life although Vt/Ttot is virtually the same in both groups of infants? (2) do aborted SIDS infants mature so that their resting respiratory pattern resemble more that of the normal infants by 3 and 4 months of life? Clearly, these questions need further evaluation.

Whether the abnormal respiratory findings in our aborted SIDS infants are secondary to the cardiorespiratory arrest with possible central nervous system injury is not known. However, Hoppenbrouwers et al. (18) have found the same increase in respiratory rate in siblings of SIDS victims that we found in aborted SIDS infants. Although both groups are considered to be at high risk for SIDS, a major difference between these two groups is that the aborted SIDS infants have sustained an episode of a cardiorespiratory arrest whereas the siblings of SIDS infants have not. This implies that the respiratory abnormalities we report in this work are probably not a result of the aborted SIDS episode itself.

HYPOTHESIS: INCREASED SYMPATHOADRENAL ACTIVITY

We have previously reported that aborted SIDS infants have a decreased RR interval (21) and a smaller QT index during sleep

QUIET SLEEP 
in the first 4 months of life (15). One possible explanation for these findings is that aborted SIDS infants have an increase in sympathoadrenal activity $(14,15,21)$. This may be, for example, in the form of increased tissue and circulating catecholamines or in the form of increased sensitivity of receptors to catecholamines. The greater increase in ventilation with $\mathrm{CO}_{2}$ found in aborted SIDS infants at 3 and 4 months of age is consistent with this hypothesis because it has been shown experimentally that acutely injected epinephrine or norepinephrine in adult man causes a greater increase in ventilation in response to $\mathrm{CO}_{2}(4,7,9)$. Additional support for this hypothesis is provided by the postmortem observation demonstrating hypertrophy of the chromaffin cell layer of the adrenal medulla in victims of SIDS (25).

Whether the increase in sympathoadrenal activity that we are hypothesizing is secondary to hypoxia or some other stimulus is not known. The noninvasive nature of our studies and the complexity of the techniques utilized precluded measuring arterial blood gases during these ventilatory studies. Several previous postmortem studies have led to the hypothesis that SIDS infants had sustained a chronic hypoxic insult before death $(24,29,33)$. To our knowledge, however, actual oxygen desaturation has not been documented and no direct evidence of hypoxemia (i.e., decreased $\mathrm{PaO}_{2}$ ) exists in these infants.

\section{REFERENCES AND NOTES}

1. Anders, T. Emde R, and Parmelee, A : A Manual of Standardized Terminology, Techniques and Criteria for Scoring of States of Sleep and Wakefulness in Newborn Infants. (BRI Publications, Los Angeles, 1971).

2. Anthonisen, N. R., and Dhingra, S.: Ventilatory response to low levels of $\mathrm{CO}_{2}$. Respir. Physiol., 32: 335 (1978).

3. Askanazi, J., Silverberg, P. A., Foster, R. J., Hyman, A. I., Milic-Emili, J., and Kinney, J. M.: Effects of respiratory apparatus on breathing pattern. J. Appl. Physiol., 48 : 577 (1980)

4. Barcroft, H., Basynayake, V., Celander, O., Cobbold, A. F., Cunningham, D. J. C., Jukes, M. G. M., and Young. I. M.: The effect of carbon dioxide on the respiratory response to noradrenaline in man. J. Physiol. (Lond.), 137: 365 (1957).

5. Birnbaum, Z. W.: On a use of the Mann-Whitney statistic. Proc. Third Berkeley Symp. Math. Statist. Probability, 1: 13 (1966).

6. Brownlee, K. A.: Statistical Theory and Methodology in Science and Engineering, p. 299 (John Wiley \& Sons, Inc., New York, 1960).

7. Cunningham, D. J. C., Hey, E. N., and Lloyd, B. B.: The effect of intravenous infusion of noradrenaline on the respiratory response to carbon dioxide in man. Q. J. Exp. Physiol. Cogn. Med. Sci., 43: 394 (1958)

8. Dejours, P., Puccinelli, R., Armand, J., and Dicharry, M.: Concept and measurement of ventilatory sensitivity to carbon dioxide. J. Appl. Physiol., 20: 890 (1965).

9. Dittrichova, J.: The development of sleep in infancy. J. Appl. Physiol., 21: 1243 (1966).

10. Drorbaugh, J. E., and Fenn, W. O.: A barometric method for measuring ventilation in newborn infants. Pediatrics, 16: 81 (1955).

11. Epstein, M. A., and Epstein, R. A.: A theoretical analysis of the barometric method for measurement of tidal volume. Respir. Physiol., 32: 105 (1978).
12. Gilbert, R., Auchincloss, J. H., Brodsky, J., and Boden, W.: Changes in tidal volume, frequency and ventilation induced by their measurement. J. Appl. Physiol., 33: 252 (1974).

13. Guz, A., Noble, M. I. M., Widdicombbe, J., Trenchard, D., and Mushin, W. W. The effect of bilaterial block of vagus and glossopharyngeal nerves on the ventilatory response to $\mathrm{CO}_{2}$ of conscious man. Respir. Physiol., I: 206 (1966).

14. Haddad, G. G., Epstein, R. A., Epstein, M. A. F., Leistner, H. L., Marino, P. A. and Mellins, R. B.: Maturation of ventilation and ventilatory pattern in normal sleeping infants. J. Appl. Physiol., 46: 998 (1979).

15. Haddad, G. G., Epstein, M. A. F., Epstein, R. A., Mazza, N. M., Mellins, R. B. and Krongrad, E.: The QT interval in aborted sudden infant death syndrome infants. Pediatr. Res., 13: 136 (1979).

16. Haddad, G. G., Leistner, H. L., Epstein, R. A., Epstein, M. A. F., Grodin, W. K. and Mellins, R. B.: $\mathrm{CO}_{2}$-induced changes in ventilation and ventilatory pattern in normal sleeping infants. J. Appl. Physiol.. 48: 684 (1980).

17. Hollander, M., and Wolfe, D. A.: Nonparametric Statistical Methods. pp. 76 (John Wiley \& Sons, Inc., New York, 1973).

18. Hoppenbrouwers, T., Hodgman, J. E., Harper, R. M., McGinty, D. J., and Sterman, M. B.: Respiratory rates and apnea in infants at high and low risk for sudden infant death syndrome (SIDS). Clin. Res., 25: 189A (1977).

19. Joels, N., and White H.: The contribution of the arterial chemoreceptors to the stimulation of respiration by adrenaline and noradrenaline in the cat. $\mathrm{J}$ Physiol. (Lond.), 197: 1 (1968).

20. Lehmann, E. L., Consistency and unbiasedness of certain nonparametric tests. Ann. Math. Statist., 22: 165 (1951)

21. Leistner, H. L., Haddad, G. G., Epstein, R. A., Lai. T. L., Epstein. M. A. F., and Mellins, R. B.: Heart rate and heart rate variability during sleep in aborted sudden infant death syndrome. J. Pediatr., 97: 51 (1980).

22. Marsland, D. W.. Callahan, B. J., and Shannon. D. C.: The afferent vagus and regulation of breathing in response to inhaled $\mathrm{CO}_{2}$ in awake newborn lambs. Biol. Neonate, 27: 102 (1975).

23. Naeye, R. L.: Pulmonary arterial abnormalities in the sudden-infant-deathsyndrome. N. Engl. J. Med., 289: 1167 (1973).

24. Naeye, R. L.: Hypoxemia and the sudden infant death syndrome. Science (Wash. D. C.), 186: 837 (1974).

25. Naeye, R. L.: Brain stem and adrenal abnormalities in the sudden-infant-death syndrome. Am. J. Clin. Pathol., 66: 526 (1976).

26. Shannon, D. C., Kelly, D. H., and O'Connell, K.: Abnormal regulation of ventilation in infants at risk for sudden infant death syndrome. N. Engl. J. Med., 297: 747 (1977).

27. Tooley, W. H.: Sudden infant death syndrome. Am. Rev. Respir. Dis., 112: 157 (1975)

28. Weitzman, E. D., and Graziani, L.: Sleep and the sudden infant death syndrome: a new hypothesis. Adv. Sleep Res., 1: 327 (1974).

29. Williams, A., Vawter, G., and Reid, L.: Increased pulmonary artery muscularity in victims of the sudden infant death syndrome (S.I.D.S.). Am. Rev. Respir. Dis., 117: 412 (1978)

30. Wolfe, D. A., and Hogg, R. V.: On constructing statistics and reporting data. Am. Statistician, 25: 27 (1971).

31. The authors thank Drs. Ralph A. Epstein and Mary Anne F. Epstein for their helpful suggestions and criticisms during the preparation of the manuscript.

32. Requests for reprints should be addressed to: G. G. Haddad, M. D., Pediatric Pulmonary Division, Columbia-Presbyterian Medical Center, 622 West 168th Street, New York. NY 10032 (USA).

33. This research was supported by National Institutes of Health Grants HD 08297 HL 06012, HL 07421, and RR 00645, Contract 52856 , and a grant from the Parker B. Francis Foundation.

34. Received for publication August 13, 1980

35. Accepted for publication October 1, 1980 